\title{
Nonuniformity Effect of Surface-Nanocrystalline Materials in Nanoindentation Test
}

\author{
Yueguang Wei, Xiaoliang Chen, Siqi Shu, E Chen Zhu \\ LNM, Institute of Mechanics, Chinese Academy of Sciences, Beijing 100080, China
}

\begin{abstract}
In the present research, microstructures of the surface-nanocrystalline Al alloy material are observed and measured based on the transmission electron microscopy (TEM) technique, and the corresponding mechanical behaviors are investigated experimentally and theoretically. In the experimental research, the nanoindentation test method is used, and the load and microhardness curves are measured, which strongly depend on the grain size and grain size nonuniformity. Two kinds of the nanoindentation test methods are adopted: the randomly selected loading point method and the continuous stiffness method. In the theoretical modeling, based on the microstructure characteristics of the surface-nanocrystalline Al alloy material, a dislocation pile-up model considering the grain size effect and based on the Mott theory is presented and used. The hardness-indent depth curves are predicted and modeled.
\end{abstract}

\section{KEY WORDS}

surface-nanocrystalline Al alloy, microstructure, TEM observation, nanoindentation test, nonuniformity effect

*Address all correspondence to ywei@LNM.imech.ac.cn 


\section{INTRODUCTION}

Recent studies have shown that high-strength, nanostructured materials can be fabricated by using some special techniques. For example, by using a severe plastic deformation (SPD) method, one can fabricate a kind of the bulk nanocrystalline (NC) materials [1-4] or can obtain a surface-nanocrystalline (SNC) layer near the surface of a conventional material, and the material mechanical behaviors can be improved considerably [5,6]. Usually, the adopted SPD methods mainly include the large torsion method [1], the large pressing method [4], and the ultrasonic shot peening (USP) method [5,6], etc. The microstructure features of both the nanostructured bulk materials and the SNC materials have been observed and measured widely in previous research [1-6]. Recent investigations have displayed that the regular microstructures exist within the SNC surface layer, and a representative fundamental cell size of the microstructure varies from tens to hundreds of nanometers, even to microns. The mechanical behaviors of the NC materials have been studied extensively in the last several years. Besides the research mentioned above [1-6], a lot of studies have covered a wide range of the NC material behaviors, such as the grain boundary behavior and plasticity in the NC Ni [7], the compressive behaviors of the $\mathrm{NC} \mathrm{Al}$ alloy [8], the surface roughness effect on the hardness of the $\mathrm{NC} \mathrm{Al}$ alloy [9], the grain rotation model based on a nine-grain cluster mechanism for the NC copper [10], the strain rate sensitivity in the NC Ni [11], the formed nanometer crystal grain due to indented for a bulk amorphous metal alloy [12], the tensile behaviors of the NC electrodeposited Ni [13], the HallPetch relation of the NC grain size effects [1416], and the high tensile ductility and the grain size nonuniform effect of the NC copper [17]. It is well known that below the micron scale, materials tend to display the strong size effect. On the size effect, a lot of research is focused on measuring and characterizing the load displacement and hardness displacement relations for single-crystal or coarse-grained metals based on the nanoindentation tests, such as the work by Ma and Clarke [18], Nix and Gao [19], McElhaney et al. [20], Wei et al. [21], Zhang et al. [22], etc. These investigations have showed that as the indent depth decreases, the measured hardness curve displays an increasing trend, the so-called indentation size effect. The size effect was often described by using the strain gradient theories [23-28] and the dislocation density theory $[9,19,29]$ as well as the discrete dislocation theory [30]. Many modeling results were quite consistent with the experimentally measured results. However, the cases involving both the NC and SNC materials are quite different from the cases of both the single-crystal and the coarse-grained materials. To the NC or SNC materials, besides the size effect, an effect of both the crystal grain size and grain shape distributions should be considered because for NC materials the grain size is comparable to the material length scale. In the previous research of the first author on the nanopolycrystal Al and the thin film/substrate system [31], the effect of both the crystal grain size and the shape distribution was called the "geometrical effect" to distinguish it from the size effect described only by the microscale parameter of the strain gradient theories. On the basis of the microstructure cell model and the strain gradient plasticity theory, the size effect and geometrical effect have been studied. Through comparing the predicted results with experimental results, the effects of both grain size and the microstructure characteristics on the microscale parameter of the strain gradient theory have been studied [31]. Besides the factors mentioned above, grain size nonuniformity is another important factor in the nanocrystalline materials that has a considerable influence on the mechanical behaviors. Some research shows that this factor 
plays a positive role in increasing the strength and ductility of the nanocrystalline materials [17].

In the present research, first, through observation and measurement using transmission electron microscopy (TEM), microstructure features in the SNC Al alloy LC4 worked by USP method will be examined; second, the mechanics behavior of the $\mathrm{SNC} \mathrm{Al} \mathrm{alloy} \mathrm{material} \mathrm{will} \mathrm{be}$ studied experimentally and theoretically based on the nanoindentation experiments. In the experimental research the specimens will be designed and prepared according to the microstructure features. The load- and hardnessdepth curves will be measured by using both the randomly selected loading point method and the continuous stiffness method. In theoretical research; using the Mott dislocation pileup theory [32], a model based on a dislocation mechanism constrained by grain boundaries will be presented and used to model the nanoindentation experiments for the $\mathrm{NC} \mathrm{Al} \mathrm{al-}$ loy material. The material hardness curves will be predicted. Finally, through experimental research and the theoretical simulation and analysis for the SNC Al alloy material, limitations of the experimental research and theoretical model and further work will be discussed.

\section{THE SNC MATERIALS}

The procedures of the experimental specimen preparations used here are similar to those shown by Wu et al. [6] for another material $\mathrm{Al}$ alloy 7075. A brief description of the procedures is given.

\subsection{Material}

The experimental material was a high-purity Al alloy LC4, with a composition (wt \%) of $2.5 \mathrm{Mg}$, $5.6 \mathrm{Zn}, 0.4 \mathrm{Mn}$, and $1.8 \mathrm{Cu}$, balance Al. A commercially available plate was cut into pieces 100 $\times 100 \times 6 \mathrm{~mm}^{3}$ in dimension. A smooth surface finish was attained on the faces by polishing on $800-1200$ grade $\mathrm{SiC}$ papers. Microscopic examination revealed an initial grain size of the order of $\sim 80 \mu \mathrm{m}$.

\subsection{The USP Technique}

The principle of the USP technique was described by $\mathrm{Lu}$ and $\mathrm{Lu}$ [5]. A brief description is given here as follows. Referring to Fig. 1(a), a high-energy ultrasonic generator with a high frequency $(45 \mathrm{kHz})$ vibrated the reflecting chamber, where the stainless steel shots of $7.45 \mathrm{~mm}$ diameter resonated. The shots then performed repetitive, high-speed, and multidirectional impacts onto the surface of the sample. As a result, severe plastic strains were imparted into the surface by striking loading. The USP processing was conducted under vacuum at room temperature for $15 \mathrm{~min}$. Through the USP technique treatment the nanometerscale crystal grains were formed near the NC surface, as shown in Figs. 1(b) and 1(c), and the grain sizes change with the distance away from the NC (striking) surface in a gradient law. Near the NC surface, nanometer-sized grains are formed, and at a distance far away from the NC surface, for instance, for distance $D>$ $80 \mu \mathrm{m}$, the grain size keeps the original coarse grain size. The grain sizes change with the distance away from the NC surface.

\section{TEM OBSERVATION AND MEASUREMENT FOR SNC AL ALLOY}

\subsection{Grain Size Distribution}

The TEM measurement of crystal grain size was performed for the SNC Al alloy. Pictures were taken at several depths away from the NC surface, and the quantities and distributions of crystal grain sizes at each depth were counted, 


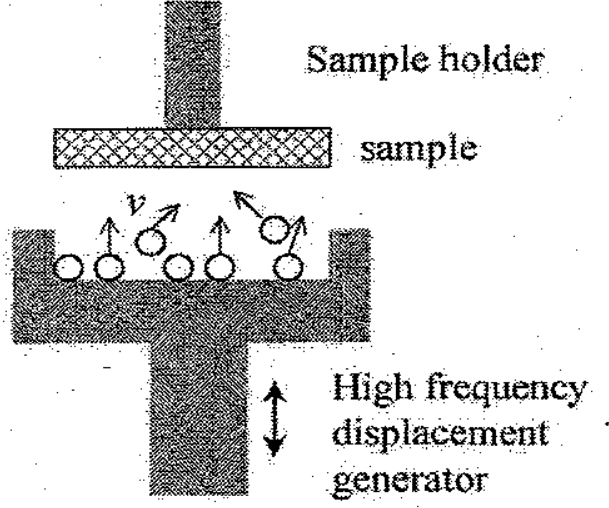

(a) Nanocrystallization principle.

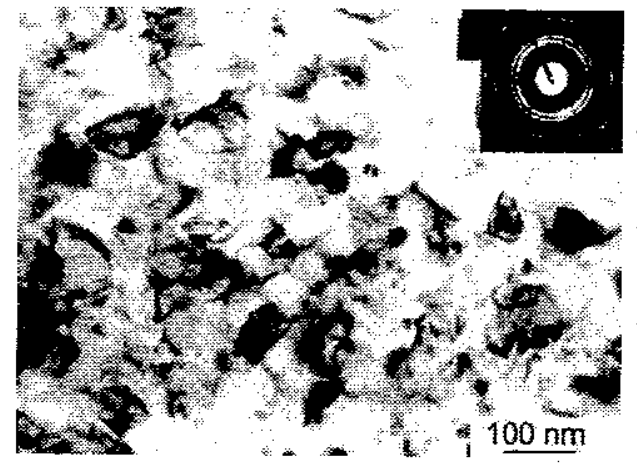

(b) The formed nanocrystal grain. $\mathrm{D}=20$ micron.

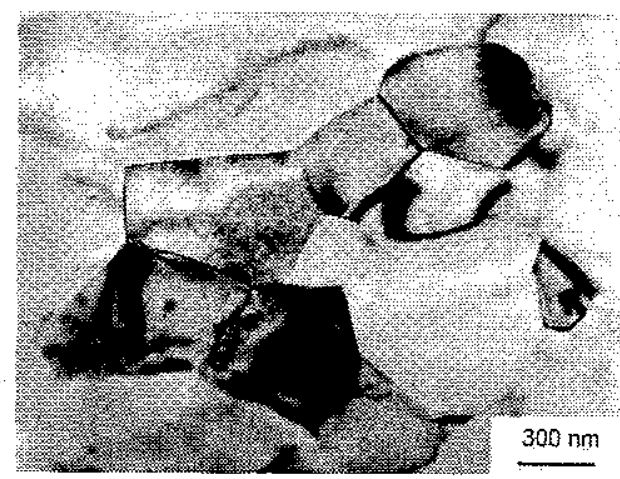

(c) Grain size nonuniformity. $\mathrm{D}=35$ micron.

FIGURE 1. Surface-nanocrystallization principle: (a) the formed nanocrystal grains and (b) the grain size nonuniformity

as shown in Figs. 2(a) and 2(b) for two distance ranges, $D=0 \sim 10 \mu \mathrm{m}$ and $D=15 \sim 20 \mu \mathrm{m}$ away from the NC surface. Fig. 2(c) shows the relationship of the statistically averaged grain size changing with distance away from the NC surface. According to the TEM observation and measurement, when the distance is larger than about $80 \mu \mathrm{m}$, the NC effect is very weak, and the original coarse grain size is kept $(80 \mu \mathrm{m})$. From Fig. 2(c), within a surface layer of $\sim 30 \mu \mathrm{m}$ thickness, the grains are nanocrystallized remarkably (grain size < $100 \mathrm{~nm}$ ).

\subsection{TEM Observation of the Grain Size Nonuniformity}

In order to investigate the nanocrystallization mechanism of the SNC Al alloy, a series of the TEM micrographs are taken for a series of distances of loading point to the NC surface: $50 \mu \mathrm{m}, 40 \mu \mathrm{m}, 35 \mu \mathrm{m}, 25 \mu \mathrm{m}$, and $15 \mu \mathrm{m}$, respectively. These TEM micrographs record the microstructure features of the SNC Al alloy corresponding to the given distances. A common feature displayed in these micrographs is that 


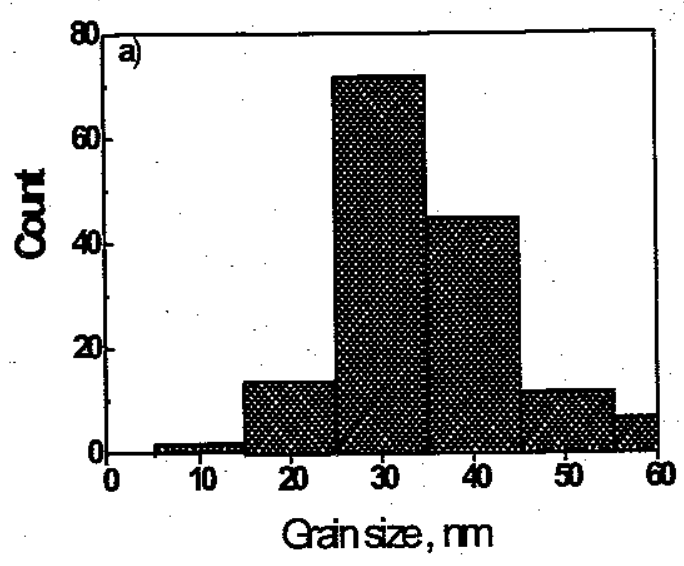

(a) $\mathrm{D}=010$ micron

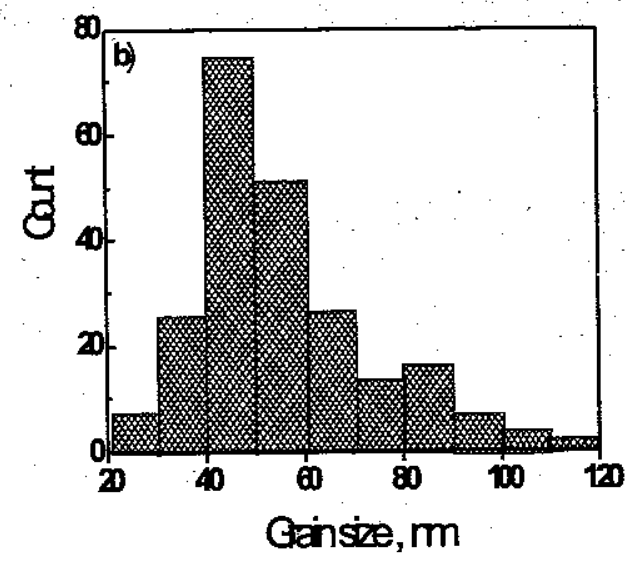

(b) $\mathrm{D}=1520$ micron

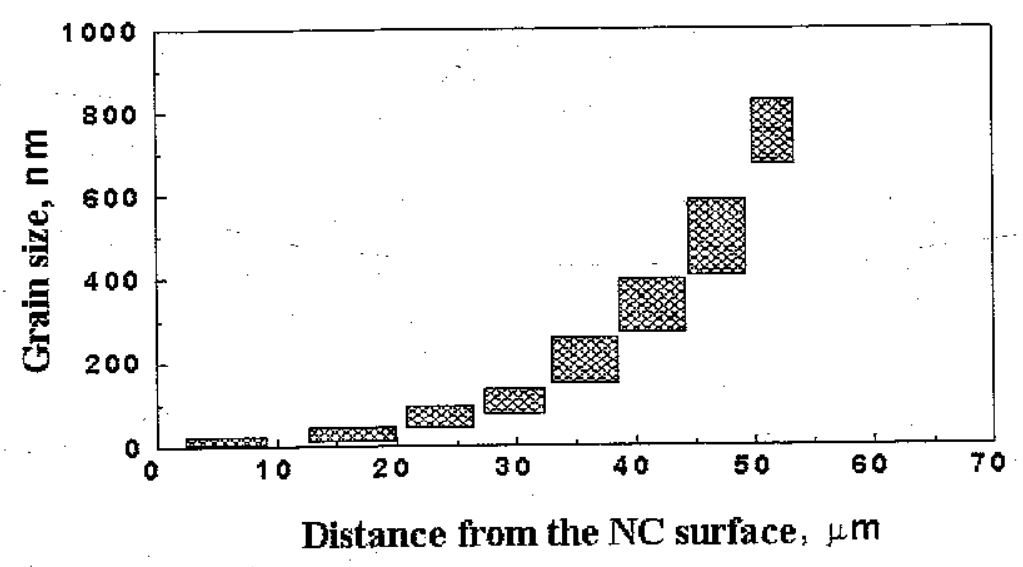

(c) Grain size distribution

FIGURE 2. Statistically averaging grain sizes changing with the distance $(D)$ away from the nanocrystalline (NC) surface of Al alloy material. Relationships between $(a, b)$ grain counts and grain sizes and (c) the grain size distribution versus distance $D$

grain size is nonuniform, as shown in Fig. 1(c) for $D=35 \mathrm{\mu m}$. There is a considerable deviation of the grain sizes between the maximum and minimum grain size. The measured microhardness should be sensitive to the grain size nonuniformity.

\section{MICROHARDNESS OF THE SNC AL ALLOY MATERIAL}

In the measurement of the microhardness the indented direction is perpendicular to the $\mathrm{NC}$ surface. The indented surfaces of specimens are 
taken at a series of distances $(D)$ away from the NC surface, corresponding to a series of the different, average grain sizes. In order to clearly describe the problem, during the nanoindentation test, by using the randomly selected loading point method, we limit the maximum indented depth through the condition that it must be smaller than the grain size. Both the randomly selected loading point method and the continuous stiffness method are adopted.

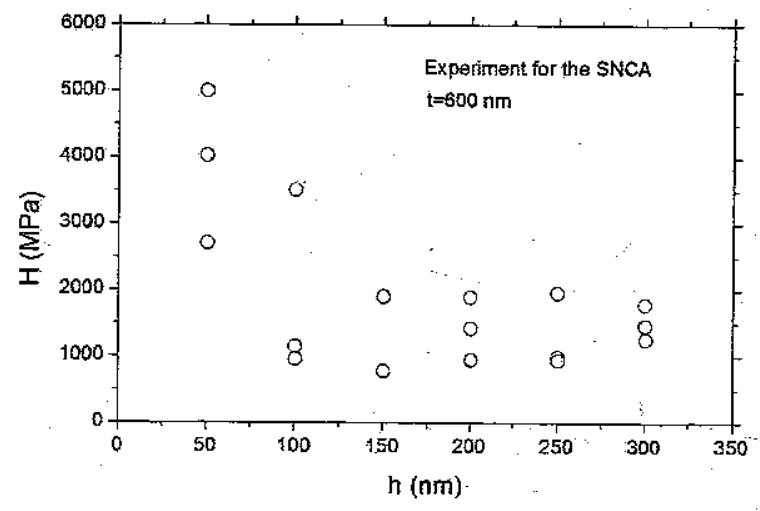

(a) $\mathrm{D}=45$ micron.

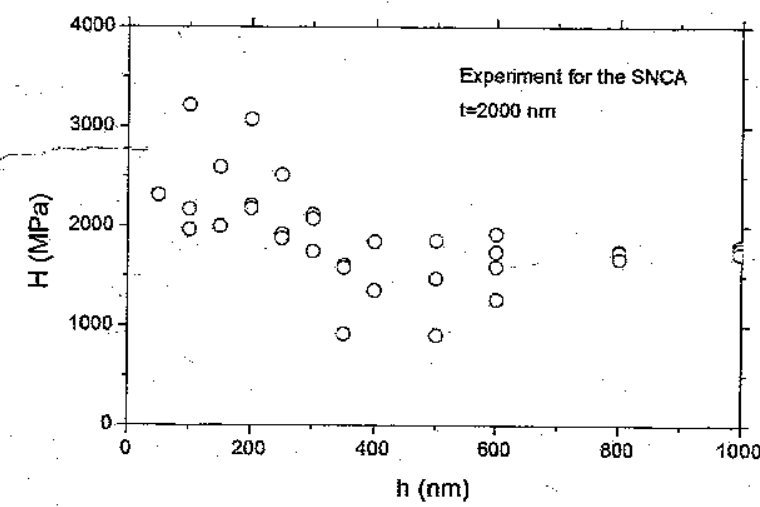

(c) $\mathrm{D}=65$ micron.

\subsection{The Randomly Selected Loading Point Method}

By randomly selecting the loading points on the specimen surface and carrying out the nanoindentation experiments, hardness-depth relations are measured, as given in Figs. 3(a)-3(d). The hardness results for several crystal grain sizes, $0.6 \mu \mathrm{m}, 1 \mu \mathrm{m}$, and $2 \mu \mathrm{m}$; as well as for single-crystal $\mathrm{Al}$ are shown, respectively. The

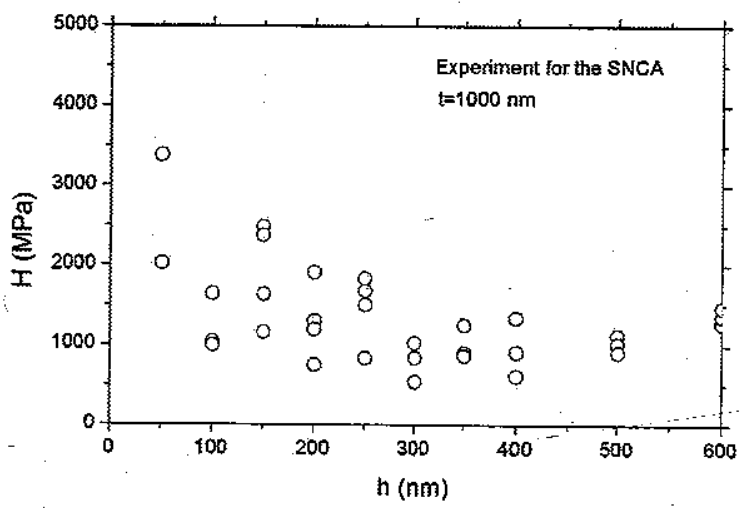

(b) $\mathrm{D}=55$ micron.

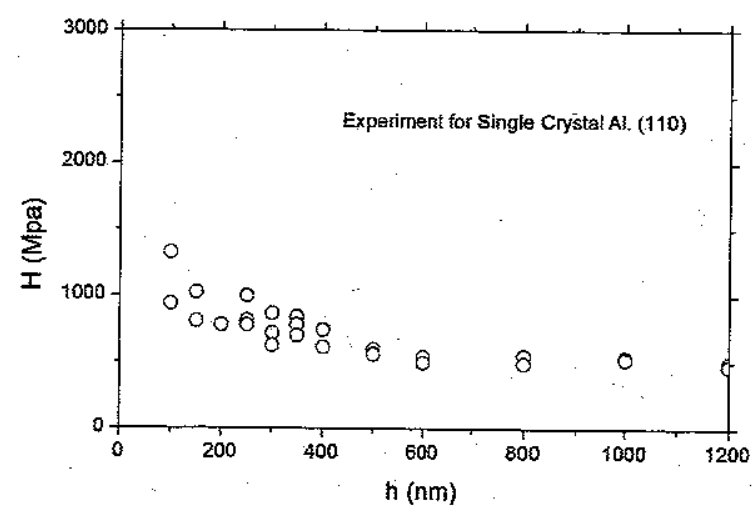

(d) Single crystal Al.

FIGURE 3. Hardness-depth data relations for $(a-c)$ three grain size cases and (d) the single crystal Al based on the randomly selected loading point method 
former three specimens correspond to the distances $D=45,55$, and $60 \mu \mathrm{m}$ away from the NC surface, respectively. The results show a feature of the hardness variation with the indent depth that when the indent depth is smaller than about a third of the grain size, a strong size effect is displayed; that is; as the indent depth increases, the hardness first decreases and passes through a lower limit value at about a third of the grain size, then increases as the plastic zone extends and is constrained by the grain boundaries. Through comparing the results shown in Figs. 3(a), 3(b), 3(c), and 3(d) for different grain sizes one can find that with decreasing grain size, the hardness increases. The hardness-depth data relation is a discrete strip with a big discrete width. For the singlecrystal case the hardness decreases and asymptotes to a conventional value as indent depth increases infinitely; the curve feature of the hardness variation with the indent depth is in " $\mathrm{L}$ " form, and the width of the hardness-depth discrete strip is much smaller than that of the NC material. It is readily understood that the big discrete hardness-depth relation for the NC material is mainly caused by the grain size nonuniformity effect. It is worth noting that for the NC materials, in the nanoindentation experiment, the indenter tip is probably located at a grain boundary region, not at a desired grain region, and in this case the measured experimental data may also have a certain deviation from the main trend of the experimental results. However, for $t>600 \mathrm{~nm}$, there is a great possibility of the indenter tip being located at the grain region because the grain region is much larger than the grain boundary region.

\subsection{The Continuous Stiffness Method}

In the present research the indent loading points were randomly selected at the distance of $30 \mu \mathrm{m}$ away from the NC surface, where the average grain size is about $200 \mathrm{~nm}$ from the
TEM measurement. Indented direction was in the normal direction of the NC surface.

Figure 4 shows the measured load- and hardness-depth curves for several selected. loading points. From Fig. 4(a) the load-depth curves seem to have a good repeat property. Several curves based on the several load points seem close to each other. However, for the hardness curves from Figs. 4(b) and 4(c), there exists a big deviation, and the results are strongly dependent on the loading points. The hardness curves are discrete mainly due to the grain size nonuniformity effect (referring to Fig. 1(c)). In Figs. 4(b) and 4(c), corresponding to the submicron-scale grain sizes, owing to the grain size nonuniformity effect, the differences between the measured hardness values based on different load points are big, and the differences decrease with increasing indent depth.

\section{MECHANISM CHARACTERIZATION AND ANALYSIS}

It is well known that the materials display a strong size effect in the submicron or nanometer scale. A lot of research discusses indentation size effects in this length scale region. Several theories concerning size effect characterizations were presented and used previously, such as the strain gradient theories [23-28], dislocation density theory $[9,19,29]$, and discrete dislocation theory [30]. The indentation size effects for single-crystal materials and for coarsegrained materials have been widely studied in the last several years; however, for the NC and SNC materials, these kinds of studies are very few. For the NC or SNC materials, besides the conventional size effect usually described by the length parameters contained in the scale theories, such as the strain gradient plasticity theory, there exists an additional length scale, grain size, which is small and comparable to the material length scale parameter. The studies for the combination effects are of important signifi- 


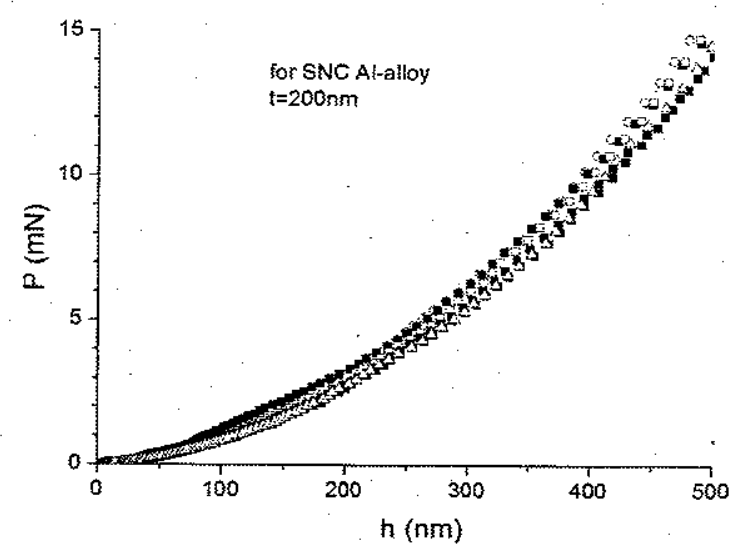

(a)

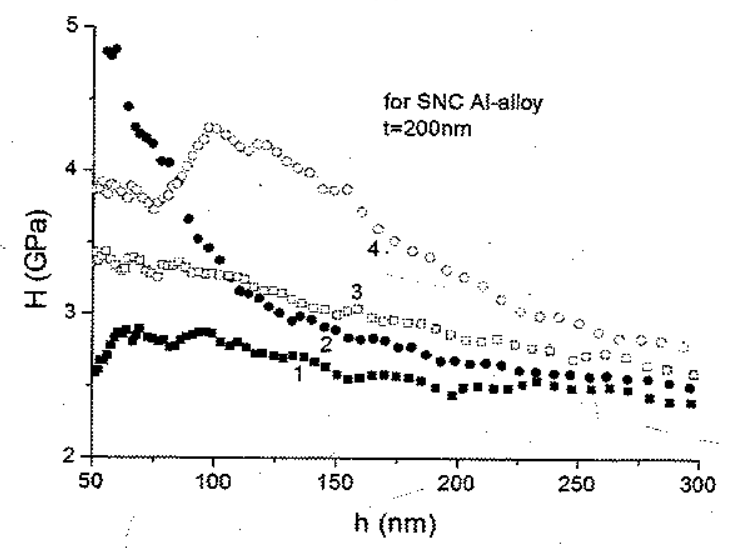

(b)

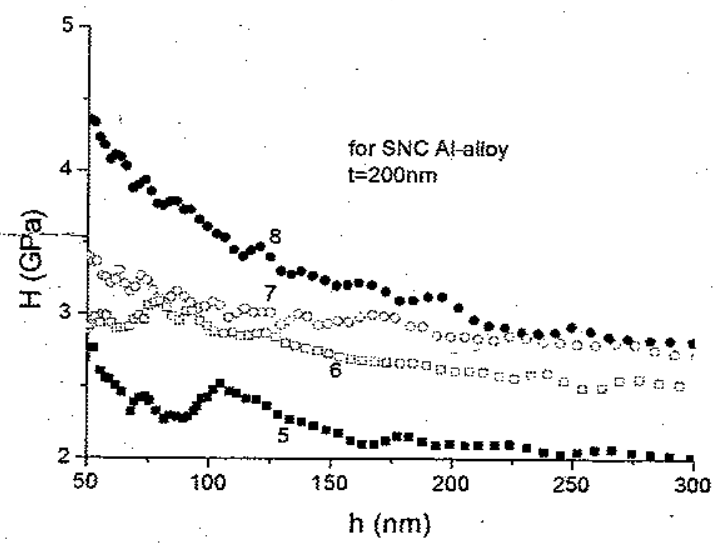

(c)

FIGURE 4. Variations of load and microhardness with indent depth for SNC Al alloy material. (a) Load-indent depth curves for several load points. Hardness-indent depth curves for different load points: (b) for one group of four load points and (c) for another group of four load points cance to understanding the mechanical properties of the nanometer materials. It is worth noting that the dislocation density model based on the Taylor theory has been widely and successfully used to describe the indentation size effect in the last several years for both the singlecrystal and coarse-grained metals, such as by Nix and Gao [19], Gao et al. [24], Wei et al. [9], and Swadener et al. [29]; however, for the NC and SNC materials, since the grain size is so small that it is comparable to the characteristic length scale of dislocation density, the grain boundary constraint is considerable, and the dislocation pileup phenomenon is prevalent. Therefore the dislocation density model based on the Taylor theory fails to describe the case. In the present research the dislocation density model based on the Mott theory [32] is used to model the mechanical behaviors of the SNC materials, such as the load- and hardnessdepth curve behaviors shown in Section 4.

\subsection{Dislocation Density Model Based on the Taylor Theory}

A brief review of the dislocation density model based on the Taylor theory is given as follows. For a nanoindentation test problem the dislocation density is nonuniform, and it can be divided into two parts: the statistically stored dislocation density and the geometrically necessary dislocation density $[33,34]$. In this case the dislocation density depends not only on the exerted load, but also on the microstructure geometrical parameters. The geometrically necessary dislocation density depends on the geometrical parameters. Through constructing a deformation-permitted dislocation mechanism, Nix and Gao [19] arrived at a simple relation between the total dislocation density and the indent depth $h, \rho_{T}=B\left(1+h^{*} / h\right)$. Putting together the related relations, such as the Taylor model relation, the Mises flow theory of plas- 
ticity, the Tabor factor relation, and the above dislocation density relation, we have

$$
\begin{array}{ll}
\tau=\alpha \mu b \sqrt{\rho_{T}}, & \rho_{T}=B\left(1+\frac{h^{*}}{h}\right), \\
\sigma=\sqrt{3} \tau, & H=3 \sigma .
\end{array}
$$

where $\tau$ and $\sigma$ are the shear flow stress and the Mises effective flow stress, respectively, $\mu$ is shear modulus, $b$ is Burger's vector, $\alpha$ is a geometrical constant with a value of about 0.3 , and $B$ and $h^{*}$ are constants to be determined in the nanoindentation test. $B$ is the statistically stored dislocation density, and $h^{*}$ is a characteristic length, characterizing a strength of the geometrically necessary dislocation density, related to the strain gradient dominated zone size [9]. For $h<h^{*}$ the geometrically necessary dislocation density prevails; otherwise, the statistically stored dislocation density prevails. From Eq. (1), Nix and Gao [19] derived

$$
H=H_{0} \sqrt{1+\frac{h^{*}}{h}}
$$

where

$$
H_{0}=3 \sqrt{3} \alpha \mu b \sqrt{B}
$$

$H_{0}$ is the macroscale hardness without considering the size effects and can be determined through using Eq. (2) to model the experimental hardness curve for a deep indentation case, i.e., for a large value of $h$. The hardnessdepth relationship based on the Taylor theory. (Eq. (2)) is simple and effective for describing the case without considering the dislocation pileup. There are two parameters in the above model, $H_{0}$ and $h^{*}$, by which the hardness-depth relationship for single-crystal metals and for coarse-grained metals can be effectively characterized. When the effect of the geometrically necessary dislocation density can be neglected, or for a deep indentation problem (small $h^{*}$ or large $h$ ), the hardness is a constant, $H_{0}$, which is only related to the macroscale parameters of material [35].

\subsection{Dislocation Density Model Based on the Mott Theory}

For a NC material the grain size is comparable to the characteristic length scale of dislocation density, and the grain boundary constraint effects are considerable. Within an indented grain region, with increasing load the dislocations nucleate, move, and pile up near grain boundaries. According to Mott theory [32], the corresponding shear flow stress within a material inside can be described as follows:

$$
\tau=\frac{1}{2 \pi} \mu(n b) \sqrt{\rho_{T}}
$$

where $n$ is the number of the pile-up dislocations along a single-edge dislocation plane. Variable $\rho_{T}$ is the total dislocation density of the superdislocation with the Burgers vector $n b$. Similarly, $\rho_{T}$ is divided into the statistically stored dislocation density and the geometrically necessary dislocation density. In order to find the geometrically necessary dislocation density, we present and adopt a model here to describe the deformation mechanism of the superdislocations in a nanoindentation test, as shown in Fig. 5, for simplicity assuming that the indenter tip will not penetrate the grain boundaries. In Fig. 5, $t$ is the representative grain size in the indenting direction. Noting that the volume occupied by the superdislocations is assumed to be a part of the semisphere due to the grain boundary constraint, similar to the derivation of Nix and Gao [19], one can arrive at

$$
H=\bar{H}_{0} \sqrt{1+\left(\frac{h^{*}}{t}\right) F\left(\frac{h}{t}, \theta\right)}
$$

where

$$
\vec{H}_{0}=\frac{3 \sqrt{3}}{2 \pi} \mu(n b) \sqrt{B}
$$

and 


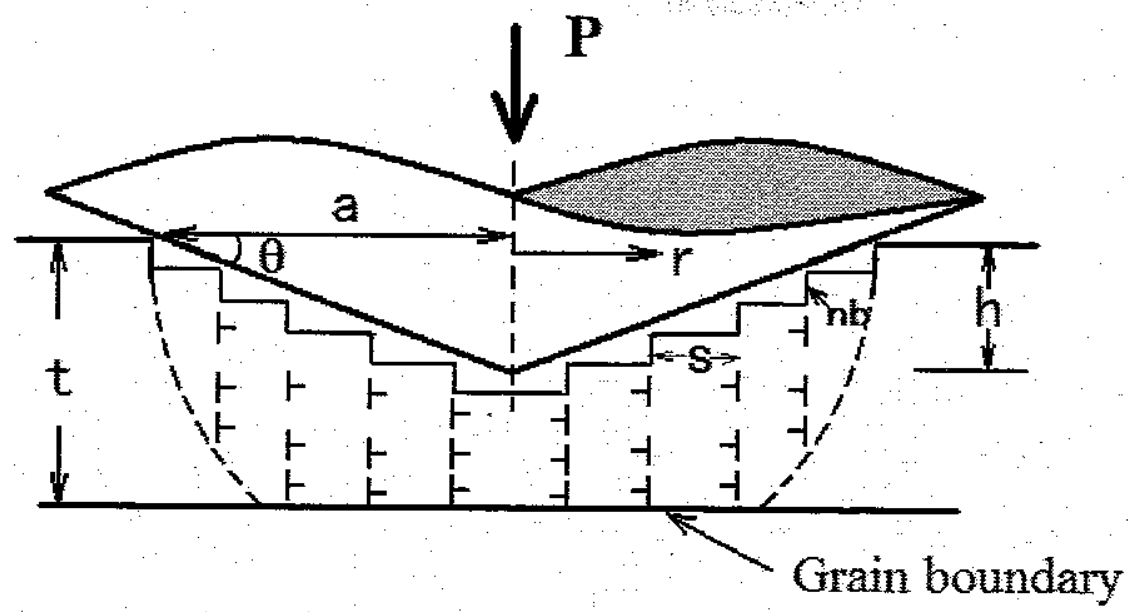

FIGURE 5. Super-dislocation deformation mechanism in nanoindentation test based on the Mott theory [32]

$$
F\left(\frac{h}{t}, \theta\right)=\left\{\begin{array}{l}
\frac{1}{\frac{h}{t}}, \frac{h}{t}<\tan \theta \\
\frac{\left(\frac{h}{t}\right)^{2}}{\left[\frac{3}{2}\left(\frac{h}{t}\right)^{2} \tan \theta-\frac{1}{2} \tan ^{3} \theta\right]}, \frac{h}{t} \geq \tan \theta
\end{array}\right.
$$

$B$ is the statistically stored dislocation density of the superdislocations. The indent angle is $\theta \approx 15^{\circ}$ for the Nanoindenter XP instrument. In derivation of Eq. (7), when $h / t \geq \tan \theta$, the adopted volume in calculating the geometrically necessary dislocation density is only a part of the semisphere, as shown in Fig. 5, instead of a complete semisphere, as adopted by $\mathrm{Nix}$ and $\mathrm{Gao}$, for applying the Taylor model to a uniform material. Comparing Eqs. (3) and (6), $\bar{H}_{0}$ is usually larger than $H_{0}$ because the piledup dislocation number, $n$, is quite a large number [32]. There are three parameters included in the dislocation density model based on the Mott theory, $\bar{H}_{0}, h^{*}$, and $t$, where $t$ is the representative grain size in the direction of indenting. Examining the presented model (Fig. 5) suggests that it may be ideal for describing the case where the grain boundary constraints on the indented grain are strong in the normal direction and weak in the tangential direction of the grain boundary. The case of weak constraint in the direction of the grain boundary seems suitable for a thin film/substrate system undergoing nanoindentation in the normal-direction of the thin film [36].

\subsection{Modeling the Microhardness of the SNC Material}

The hardness-depth relationships of the NC materials based on the present model are plotted in Fig. 6, which shows the curves of $H / \bar{H}_{0} \sim$ $h / t$ for several values of $h^{*} / t$. From Fig. 6 the hardness increases as indent depth decreases. However, as indent depth increases, the hardness-depth curves quickly tend to a stable value, described by

$$
\frac{H}{\bar{H}_{0}} \approx \sqrt{1+\frac{\left(\frac{h^{*}}{t}\right)}{\left(\frac{3}{2} \tan \theta\right)}}
$$

from Eqs. (5) and (7), instead of unity in using the Taylor theory. For comparison the Taylor theory results are also shown in Fig. 6 for several $h^{*}$ values (see dashed lines), where $t$ can be understood as any reference length. The hardness curves based on the Taylor theory quickly 


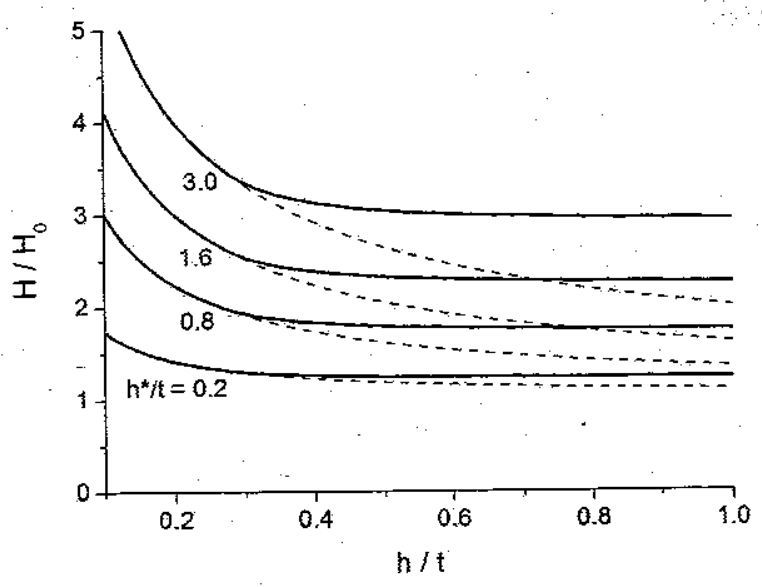

FIGURE 6. Variations of normalized hardness with the normalized indent depth for several grain size values based on the present model. Grain size as normalizing quantity. Solid lines stand for using the present model, and dashed lines stand for using the Taylor model

decrease as indent depth increases. When indent depth tends to infinity, $H / \bar{H}_{0}$ tends to unity. On the other hand, for $h^{*}$ fixed the hardness increases as grain size $(t)$ decreases.

By using the above theoretical analysis results to model the experimental results of the SNC Al alloy shown in Fig. 4, we obtain the hardness-depth curves, as shown in Fig. 7, where several parameters are taken as follows: the average grain size $t=200 \mathrm{~nm}$; length scale $h^{*}=165 \mathrm{~nm}$ (from Wei et al. [9]); and $\bar{H}_{0}=$ 1.7 GPa (the only selected parameter according to experimental curves). Comparing the modeling results shown in Fig. 7 and the experimental results shown in Fig. 4, one finds that the simple model developed in the present research can roughly capture the complicated microhardness features of the SNC Al alloy LC4.

\section{CONCLUDING REMARKS}

The Al alloy LC4 has been surfacenanocrystallized by using the USP method,

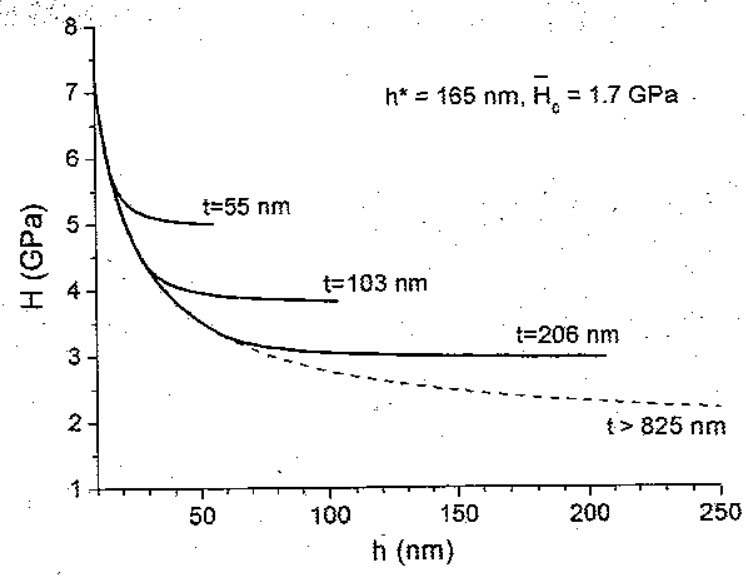

FIGURE 7. Microhardness modeling for the SNC Al alloy material by using the present model. Hardness-depth curves are grain size-sensitive

and the nanometer-sized grains have been produced within the surface layer of the specimen. The microstructure features of the $\mathrm{NC}$ region have been observed and measured by using the TEM technique. The deformation mechanism of the SNC material has been characterized and investigated based on the nanoindentation experiments and the dislocation pile-up model. Experimental results have displayed that the hardness-depth curves of the SNC material are with a strong size effect and a strong nonuniformity effect of grain size. Theoretical modeling results are consistent with the experimental results.

On the strength and hardness of the polycrystalline material, there exists a famous HallPetch relationship. According to the Hall-Petch relationship, the strength and hardness of the polycrystalline materials increase with decreasing grain size. Although the NC material is a kind of polycrystalline material, the crystal grain size of the NC materials is at the nanometer scale, so how effective is the Hall-Petch relationship in this case? To answer this question, there has been considerable research in last decade, for example, the research of Nieh 
and Wadsworth [14], Nieh and Wang [15], and Yamakov et al. [16]. A lot of research has shown that the strength and hardness of the NC materials varying with the grain size can be characterized by the Hall-Petch relationship when grain size is larger than about $20-40 \mathrm{~nm}$ and by an inverse Hall-Petch relationship when grain size is smaller than that value for the nanocrystalline metals. In the present research our attention has focused on investigating the size effect and the geometrical effect (including the grain size nonuniformity effect), and therefore we have obtained hardness information for several grain sizes of the nanocrystalline $\mathrm{Al}$ alloy when grain size is larger than $100 \mathrm{~nm}$. Owing to the limitations of the currently used the Nanoindenter XP instrument (indenter tip curvature radius is about $50 \mathrm{~nm}$ ), we cannot obtain reasonable hardness results when the grain size is too small. No matter the limitations of the Nanoindenter instrument, we can assess the Hall-Petch relationship when grain size is larger than $100 \mathrm{~nm}$. In this grain size region, obviously, the Hall-Petch relationship is reasonable.

\section{ACKNOWLEDGMENT}

This work was supported by the National Natural Science Foundation of China through Grants No. 10432050 and No. 10428207.

\section{REFERENCES}

1. Valiev, R. Z., Korznikov, A. V., and $\mathrm{Mu}^{-}$ lyukov, R. R., Structure and properties of ultrafine-grained materials produced by severe plastic deformation, Mater. Sci. Eng. A 168:141148, 1993.

2. Valiev, R. Z., Ivanisenko, Y. V., Rauch, E. F.; and Baudelet; B., Structure and deformation behavior of armco iron subjected to severe plastic deformation., Acta Mater. 44:4705-4712, 1996.
3. Valiev, R. Z., and Islamgaliev, R. K., Enhanced superplasticity of ultrafine-grained alloys processed by severe plastic deformation, Mater. Sci. Forum 304:39-46, 1999.

4. Valiev, R. Z., Islamgaliev, R. K., and Alexandrov, I. V. Bulk, nanostructured materials from severe plastic deformation, Prog. Mater. Sci. 45:103-189, 2000.

5. Lu, K., and Lu, J., Surface nanocrystallization (SNC) of metallic materials presentation of the concept behind a new approach, J. Mater. Sci. Technol. 15:193-197, 1999.

6. Wu, X., et al., Microstructure and evolution of mechanically induced ultrafine grain in surface layer of Al-alloy subjected to USSP, Acta Mater. 50:2075-2084, 2002.

7. Shan, Z., et al., Grain boundary-mediated plasticity in nanocrystalline nickel, Science 305:654$657,2004$.

8. 'Wei, Y., Zhu, C., and Wu, X., Microscale mechanics investigations of the surfacenanocrystalline Al-alloy material, Sci. China, Ser. G 47:86-100, 2004.

9. Wei, Y., Wang, X., and Zhao, M., Size effect measurement and characterization in nanoindentation test, J. Mater. Res. 19:208-217, 2004.

10. Yang, W., and Wang, H., Mechanics modeling for deformation of nano-grained metals, $J$. Mech. Phys. Solids 52:875-889, 2004.

11. Schwaiger, R., Moser, B., Dao, M., Chollacoop, N., and Suresh, S., Some critical experiments on the strain rate sensitivity of nanocrystalline nickel, Acta Mater. 51:5159-5172, 2003.

12. Kim, J., Choi, Y., Suresh, S., and Argon, A. S., Nanocrystallization during nanoindentation of a bulk amorphous metal alloy at room temperature, Science 295:654-657, 2002.

13. Torre, F., Swygenhoven, H. V.; and Victoria, M., Nanocrystalline electrodeposited Ni: microstructure and tensile properties, Acta Mater. 50:3957-3970, 2002.

14. Nieh, T. G., and Wadsworth, J., Hall-Petch relation in nanocrystalline solids, Scr. Metall. Mater. 25:955-958, 1991.

15. Nieh; T. G., and Wang, J. G., Hall-Petch relationship in nanocrystalline $\mathrm{Ni}$ and Be-B alloys, Intermetallics 13:377-385, 2005. 
16. Yamakov, V., Wolf, D., Salazar; M., Phillpot, S. R., and Gleiter, H., Length-scale effects in the nucleation of extended dislocations in nanocrystalline $\mathrm{Al}$ by molecular-dynamics simulation, Acta Mater. 49:2713-2722, 2001.

17. Wang, Y., Chen, M., Zhou, F., and Ma, E., High tensile ductility in a nanostructured metal, $\mathrm{Na}$ ture 419:912-915, 2002.

18. $\mathrm{Ma}, \mathrm{Q}$, and Clarke, D. R., Size dependent hardness of silver single crystals; J. Mater. Res. 10:853-863, 1995.

19. Nix, W. D., and Gao, H., Indentation size effects in crystalline materials: a law for strain gradient plasticity, J. Mech. Phys. Solids 46:411-425, 1998.

20. McElhaney, K. W., Vlassak, J. J., and Nix, W. D., Determination of indenter tip geometry and indentation contact area for depth-sensing indentation experiments, J. Mater. Res. 13:1300-1306, 1998

21. Wei, Y., Wang, X., Wu, X., and Bai, Y., Theoretical and experimental researches of size effect in micro-indentation test, Sci. China, Ser. A 44:74$82,2001$.

22. Zhang, T. Y., Wu, W. H., and Zhao, M. H., The role of plastic deformation of rough surfaces in the size-dependent hardness, Acta Mater. 52:57$68 ; 2004$.

23. Fleck, N. A., and Hutchinson, J. W., Strain gradient plasticity, Adv. Appl. Mech. 33:295-361, 1997.

24. Gao, H., Huang, Y., Nix, W. D., and Hutchinson, J. W., Mechanism-based strain gradient plasticity-I. Theory, J. Mech. Phys. Solids 47:1239-1263, 1999.

25. Chen, S., and Wang, T. C., A new hardening law for strain gradient plasticity, Acta Mater. 48:3997-4005, 2000.
26. Wei, Y., and Hutchinson, J. W., Steady-state crack growth and work of fracture for solids characterized by strain gradient plasticity, J. Mech. Phys. Solids 45:1253-1273, 1997.

27. Begley, M., and Hutchinson, J. W., The mechanics of size-dependent indentation, J. Mech. Phys. Solids 46:2049-2068, 1998.

28. Wei, Y., and Hutchinson, J. W., Hardness trends in micron scale indentation, J. Mech. Phys. Solids 51:2037-2056, 2003.

29. Swadener, J. G., George, E. P., and Pharr, G. M., The correlation of the indentation size effect measured with indenters of various shapes, $J$. Mech. Phys. Solids 50:681-694, 2002.

30. Cleveringa, H., Gissen, E., and Needleman, A., A discrete dislocation analysis of bending, Int. J. Plasticity 15:837-868, 1999.

31. Wei, Y., Wang, X., Zhao, M., Cheng, C. M., and Bai, Y. L., Size effect and geometrical effect of solids in micro-indentation test, Acta Mech. Sinica 19:59-70, 2003.

32. Mott, N. F., The theory of the properties of materials, Philos. Mag. 43:1151-1157, 1952.

33. Ashby, M. F., The deformation of plastically non-homogeneous material, Philos. Mag. 21:399-424, 1970.

34. Fleck, N. A., Muller, G. M., Ashby, M. F., and Hutchinson, J. W., Strain gradient plasticity theory and experiment, Acta Metall. Mater. 42:475-487, 1994.

35. Cheng, Y. T., and Cheng, C. M., Scaling relationships in conical indentation of elasticperfectly plastic solids, Int. J. Solids Struct. 36:1231-1243, 1999.

36. Kriese, M. D., Gerberich, W. W., and Moody, N. R., Quantitative adhesion measures of multilayer films: Part I: indentation mechanics, J. Mater. Res. 14:3007-3018, 1999. 